\title{
An equivalent roughness model for seabed backscattering at very high frequencies using a band-matrix approach
}

\author{
Wendelboe, Gorm; Jacobsen, Finn; Bell, Judith
}

Published in:

Acoustical Society of America. Journal

Link to article, DOI:

$10.1121 / 1.2427127$

Publication date:

2007

Document Version

Publisher's PDF, also known as Version of record

Link back to DTU Orbit

Citation (APA):

Wendelboe, G., Jacobsen, F., \& Bell, J. (2007). An equivalent roughness model for seabed backscattering at very high frequencies using a band-matrix approach. Acoustical Society of America. Journal, 121(2), 814-823. https://doi.org/10.1121/1.2427127

\section{General rights}

Copyright and moral rights for the publications made accessible in the public portal are retained by the authors and/or other copyright owners and it is a condition of accessing publications that users recognise and abide by the legal requirements associated with these rights.

- Users may download and print one copy of any publication from the public portal for the purpose of private study or research

- You may not further distribute the material or use it for any profit-making activity or commercial gain

- You may freely distribute the URL identifying the publication in the public portal 


\title{
An equivalent roughness model for seabed backscattering at very high frequencies using a band-matrix approach
}

\author{
Gorm Wendelboe ${ }^{a}$ \\ Danish Defense Research Establishment (DDRE), Ryvangs Allé 1, DK-2100 Copenhagen and Ørsted-DTU, \\ Technical University of Denmark, Ørsteds Plads, Building 348, DK-2800 Kgs. Lyngby, Denmark \\ Finn Jacobsen ${ }^{\text {b) }}$ \\ Ørsted-DTU, Technical University of Denmark, Ørsteds Plads, Building 348, DK-2800 Kgs. Lyngby, \\ Denmark \\ Judith M. Bell ${ }^{\text {c) }}$ \\ School of Engineering and Physical Sciences, Heriot-Watt University, Edinburgh, Scotland
}

(Received 10 August 2006; revised 28 November 2006; accepted 5 December 2006)

\begin{abstract}
This work concerns modeling of very high frequency $(>100 \mathrm{kHz})$ sonar images obtained from a sandy seabed. The seabed is divided into a discrete number of 1D height profiles. For each height profile the backscattered pressure is computed by an integral equation method for interface scattering between two homogeneous media as formulated by Chan [IEEE Trans. Antennas Propag. 46, 142-149 (1998)]. However, the seabed is inhomogeneous, and volume scattering is a major contributor to backscattering. The SAX99 experiments revealed that the density in the unconsolidated sediment within the first $5 \mathrm{~mm}$ exhibits a high spatial variation. For that reason, additional roughness is introduced: For each surface point a stochastic realization of the density along the vertical is generated, and the sediment depth at which the density has its maximum value will constitute the new height field value. The matrix of the full integral equation is reduced to a band matrix as the interaction between the point sources on the seabed is neglected from a certain range; this allows computations on long height profiles with lengths up to approximately $25 \mathrm{~m}$ (at $300 \mathrm{kHz}$ ). The equivalent roughness approach, combined with the band-matrix approach, agrees with SAX99 data at $300 \mathrm{kHz}$. () 2007 Acoustical Society of America. [DOI: 10.1121/1.2427127]
\end{abstract}

PACS number(s): 43.30.Gv, 43.30.Hw, 43.30.Ft [SLB]

Pages: $814-823$

\section{INTRODUCTION}

Very high frequency $(>100 \mathrm{kHz})$ sonar plays a key role for naval mine detection and identification. This work is related to mines lying on the seabed, i.e., proud mines, and the goal is modeling of high frequency sonar images. Sonar images of a sandy seabed are contaminated with clutter, a term that refers to the noisy, or unwanted, component of the received sonar signal; its strength governs the detection performance of the backscattered pressure from an object, or, say, the signal-to-reverberation ratio. Hence, clutter has a key role in manually controlled as well as automated mine detection/ identification systems since it can affect the false alarm rates. ${ }^{1}$ Clutter is the result of an oscillating pressure signal scattered back from the seabed, where a complex wave interference on the water/sediment interface and in the sediment volume occur.

Wave scattering from random rough surfaces can be modeled by a field average over an ensemble of random surfaces where the scattered acoustic power is derived analytically (see, e.g., Ref. 2 and Ref. 3, Chap. 9.3). Alternatively, the scattered pressure can be computed numerically from a stochastic realization of the rough surface, that is, a

\footnotetext{
${ }^{a)}$ Electronic mail: gw @oersted.dtu.dk

${ }^{b)}$ Electronic mail: fja@oersted.dtu.dk

${ }^{c)}$ Electronic mail: j.bell@hw.ac.uk
}

Monte Carlo approach (see e.g., Ref. 4). A combination of the average field method and the Monte Carlo approach has been applied in some sonar simulation models. ${ }^{5,6}$ However, such models generate energy-based reflectograms that do not represent the rapid phase variations obtained from real signals. The field scattered from a rough interface can be computed by the classical composite model, ${ }^{2,3}$ a combination of the Kirchhoff approximation and the small perturbation approximation, but the small slope approximation, a relatively new model, is valid for a broader range of surfaces (see, e.g., Ref. 3, Chap. 9.14 and Refs. 7 and 8). The full integral equation solution and approximate integral equation methods have been applied on 1D surfaces, see, e.g., Refs. 9-11. Meanwhile, models for interface scattering are not sufficient for acoustic field interaction with the seabed; acoustic waves penetrate into the sediment and inhomogeneities induce a scattered field. Jackson ${ }^{12-16}$ applied the small perturbation approximation for frequencies below $100 \mathrm{kHz}$, that is, for wavelengths greater than approximately $1.5 \mathrm{~cm}$; for smaller wavelengths the model can fail. ${ }^{17}$ At $140 \mathrm{kHz}$, volume scattering from strongly inhomogeneous sediments can be the dominating scattering mechanism, ${ }^{18}$ a conclusion that also may be valid for the backscattering experiment at $300 \mathrm{kHz}$ conducted at the Sediment Acoustic Experiment in 1999 (SAX99). ${ }^{19}$ Small scale measurements of the density and sound speed variability conducted at SAX99 tend to confirm that ${ }^{20}$ within the first centimeter the sediment is unconsoli- 
dated and the geoacoustical parameters exhibit a significantly higher spatial variability than in the deeper parts of the sediment.

In the model presented here the rapid phase variations are required, and a stochastic seabed realization that covers all roughness scales, i.e., a full Monte Carlo approach, will be applied. The sandy seabed is approximated by a discrete number of 1D height profiles in planes similar to the sonar beams. The height profiles are synthesized by application of seabed roughness parameters acquired from stereophotogrammetric measurements at SAX99. The scattering problem is solved by using the formulation by Chan, ${ }^{11}$ where a rough interface divides two homogeneous fluids. Volume scattering is taken into account by introducing the equivalent roughness approximation: For each surface point a stochastic realization of the density along the vertical is generated and the sediment depth at which the density has its maximum value will constitute the new height profile value. The new height profile is subsequently filtered by an AR(1)-filter in order to generate correlation along the horizontal direction. The scattering matrix is reduced to a band matrix as interactions between point sources on the seabed are neglected from a certain range, and the reduction will allow computations of long height profiles. The number of nonzero diagonals are evaluated in terms of a tradeoff between accuracy and required height profile lengths. The equivalent roughness approach combined with the band-matrix approximation is compared with SAX99 data at $300 \mathrm{kHz}$ (see Ref. 21).

All simulations are carried out with a $300-\mathrm{kHz}$ sinusoid and the speed of sound in water is assumed to be $c_{1}$ $=1500 \mathrm{~m} / \mathrm{s}$, thus the acoustic wavelength in water is $\lambda$ $=0.5 \mathrm{~cm}$.

\section{SEABED MODELING}

In this section modeling of the rough water-sediment interface as well as modeling of the density variations in the upper sediment are described. The seabed is considered as a height field; hence, the height, $h$, is a function of the ground plane coordinates, $x$ and $y$, i.e., $h=h(x, y)$. In this work, the sandy seabed is approximated by a discrete number of $1 \mathrm{D}$ height profiles in planes similar to the sonar beams. Thus, each height profile is given as

$$
h=h(x),
$$

where $x$ is the ground range coordinate along the height profile. Simulations by George ${ }^{22}$ indicate that the backscattering characteristics are independent of sonar beam width, a result that supports the height profiles approximation.

\section{A. Interface roughness}

The seabed roughness is characterized in terms of its power spectrum, which according to, e.g., Briggs et al.,$^{23}$ is given by

$$
P\left(f_{s}\right)=\frac{\phi}{f_{s}^{\gamma}},
$$

where $f_{s}$ is the spatial frequency measured in cycles/cm, $\phi$ is the spectral intercept measured in $\mathrm{cm}^{3}$, and $\gamma$ is the spectral
TABLE I. Applied interface roughness parameters.

\begin{tabular}{lccc}
\hline \hline Two-power law & $\begin{array}{c}f_{s} \\
\left(\mathrm{~cm}^{-1}\right)\end{array}$ & $\gamma$ & $\begin{array}{c}\phi \\
\left(\mathrm{cm}^{3}\right)\end{array}$ \\
\hline Large scale & $0.02-2.0$ & 3.00 & 0.00075 \\
Small scale & $2.0-4.0$ & 3.81 & 0.00131 \\
\hline \hline
\end{tabular}

exponent, which is dimensionless. Taking the logarithm on each side of Eq. (2) yields

$$
\log _{10} P\left(f_{s}\right)=\log _{10}(\phi)-\gamma \log _{10}\left(f_{s}\right),
$$

where the spectral intercept is found at $f_{s}=1$, i.e., at one cycle per centimeter. The power spectrum parameters are estimated by in situ experiments with stereophotogrammetric equipment. ${ }^{1,23-25}$ The seabed may contain several power laws distributed over the different spatial frequencies. Here, a two-power-law spectrum is used,

$$
P\left(f_{s}\right)=\left\{\begin{array}{cc}
\phi_{1} f_{s}^{-\gamma_{1}}, & f_{s}^{(\min )}<f_{s}<f_{s}^{(\mathrm{tr})}, \\
\phi_{2} f_{s}^{-\gamma_{2}}, & f_{s}^{(\mathrm{tr})}<f_{s}<f_{s}^{(\max )}, \\
0, & \text { elsewhere, }
\end{array}\right.
$$

where $\phi_{1}, \gamma_{1}$, and $\phi_{2}, \gamma_{2}$ are the spectral intercept and spectral exponent for the the first and second power laws, respectively. The minimum spatial frequency, $f_{s}^{(\min )}$, is governed by the size of the experimental area; $f_{s}^{(\mathrm{tr})^{s}}$ is the transition frequency between the two power laws; and the maximum spatial frequency, $f_{s}^{(\max )}$, is related to the resolution of the stereo-photogrammetric system.

Throughout this work $\phi_{1}, \gamma_{1}, \phi_{2}$, and $\gamma_{2}$ are based on data presented in Ref. 23 (BAMS, 5 Oct. N, Table II, p. 511). The parameters are listed in Table I. Note, the maximum spatial frequency is chosen to be $f_{s}^{(\max )}=4 \mathrm{cycles} / \mathrm{cm}$, although the value is 5 cycles/cm in Ref. 23 . This choice will become clear in the following.

The height profiles are synthesized in the frequency domain. Equation (4) is realized by suppressing frequencies below $f_{s}^{(\min )}$ by application of a tapered cosine window,

$$
W_{1}\left(f_{s}\right)=\left\{\begin{array}{cc}
\frac{1}{2}\left[1-\cos \left(\pi f_{s} / f_{s}^{(\mathrm{min})}\right)\right], & \left|f_{s}\right|<f_{s}^{(\mathrm{min})}, \\
1, & \text { elsewhere, }
\end{array}\right.
$$

and, additionally, by suppressing frequencies above $f_{s}^{(\max )}$ by application of the following tapered cosine window,

$$
W_{2}\left(f_{s}\right)=\left\{\begin{array}{cc}
\frac{1}{2}\left[1-\cos \left(\pi \frac{f_{g}-f_{s}}{f_{g}-f_{s}^{(\max )}}\right)\right], & \left|f_{s}\right|>f_{s}^{(\max )}, \\
1, & \text { elsewhere, }
\end{array}\right.
$$

where $f_{g}$ is the Nyquist frequency. The Fourier transform of the synthetic height profile, $h(x)$, is

$$
H\left(f_{s}\right)=N\left(f_{s}\right) W_{1}\left(f_{s}\right) W_{2}\left(f_{s}\right) \sqrt{P\left(f_{s}\right)},
$$

where $N\left(f_{s}\right)$ is the Fourier transform of a random Gaussian variable with unit variance. The first and second order de- 


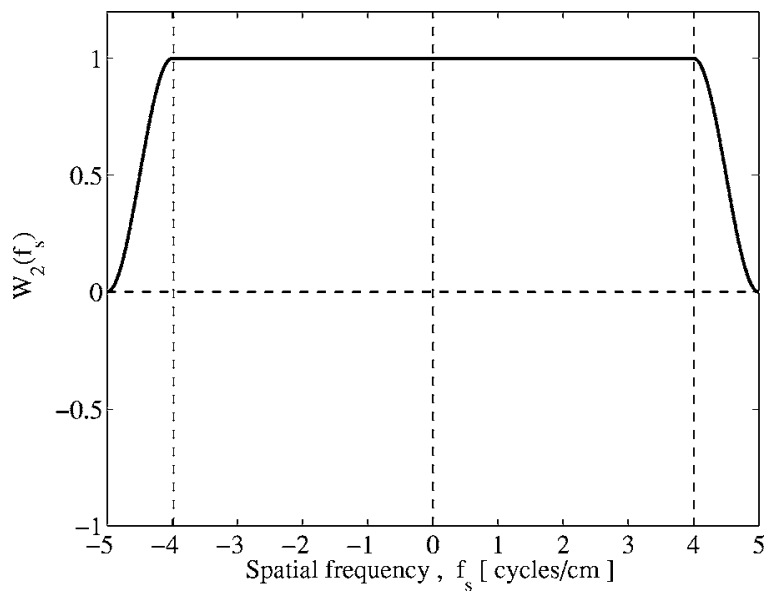

FIG. 1. The tapered cosine, frequency domain window, $W_{2}\left(f_{s}\right)$, see Eq. (6). It is applied on the surface, $h$, its derivative, $d h / d x$, and the second order derivative, $d^{2} h / d x^{2}$.

rivatives of the height profile are required in the scattering formulation presented in Sec. III. Since

$$
h(x) \leftrightarrow H\left(f_{s}\right),
$$

the surface derivatives are given by

$$
\begin{aligned}
& \frac{d h}{d x} \leftrightarrow 2 \pi i f_{s} H\left(f_{s}\right), \\
& \frac{d^{2} h}{d x^{2}} \leftrightarrow-4 \pi^{2} f_{s}^{2} H\left(f_{s}\right) .
\end{aligned}
$$

If the equidistant step range along the ground range axis (the $x$ axis) is equal to

$$
\Delta x=0.2 \lambda=0.1 \mathrm{~cm},
$$

the spatial Nyquist frequency is

$$
f_{s}^{\text {(Nyquist) }}=5 \text { cycles } / \mathrm{cm} \text {. }
$$

The Bragg spatial frequency (or wave number) for backscattering at zero grazing angle is

$$
f_{s}^{(\text {Bragg) }}=4 \text { cycles } / \mathrm{cm}
$$

(see the Appendix); it is the maximum spatial frequency of the rough surface where reinforcement of the backscattered signal can occur. For a sonar model the grazing angle may vary between, say, $\theta=10^{\circ}$ and $\theta=75^{\circ}$, and consequently, the Bragg wave numbers will approximately lie between 1 to 3.9 cycle $/ \mathrm{cm}$.

Figures 1-3 show $W_{2}\left(f_{s}\right), \quad 2 \pi i f_{s} W_{2}\left(f_{s}\right)$, and $-4 \pi^{2} f_{s}^{2} W_{2}\left(f_{s}\right)$, respectively. The tapered cosine window, $W_{2}\left(f_{s}\right)$, suppresses undesired high frequency content in $h\left(x_{n}\right)$ and its the first and second order derivative. The ground range resolution, specified in Eq. (11), has been selected such that $W_{2}\left(f_{s}\right)$ tapers off between the maximum Bragg wave number and the Nyquist frequency, and that explains why the implementation uses $f_{s}^{(\max )}=f_{s}^{(\text {Bragg })}=4$ cycles $/ \mathrm{cm}$ and not 5 cycles/cm as in Ref. 23.

In the literature $\Delta x$ may vary from $0.2 \lambda$ down to $0.05 \lambda$ [see, e.g., Ref. 4 (Appendix 1, Table 1]. Here, an analysis of convergence has shown that a resolution of $0.2 \lambda$ yields back-

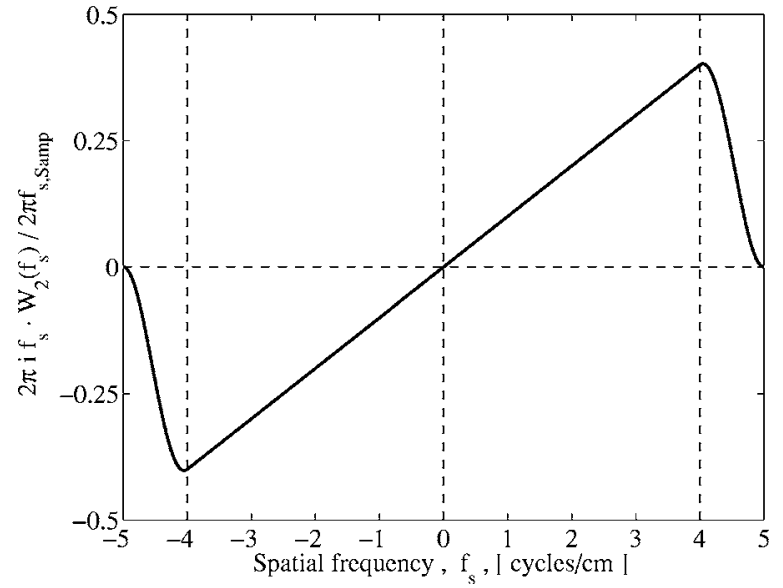

FIG. 2. Frequency domain window applied to obtain the surface first order derivative, $d h / d x$.

scattering strengths that nearly coincide with backscattering strengths obtained at a resolution of $0.1 \lambda$. Thus, a resolution of $\Delta x=0.2 \lambda$ is applied. The distance between adjacent points on the rough surface, i.e., the arc-length of the $n$th height profile sample, $\Delta s\left(x_{n}\right)$, depends on the magnitude of the surface derivative, $\gamma\left(x_{n}\right)$, by ${ }^{9}$

$$
\Delta s\left(x_{n}\right)=\gamma\left(x_{n}\right) \Delta x,
$$

where $x_{n}$ is $n$th ground range sample, and where the magnitude of the surface derivative is given by

$$
\gamma^{2}\left(x_{n}\right)=1+\left.\left(\frac{d h}{d x}\right)^{2}\right|_{x=x_{n}} .
$$

For very steep height variations in the profile the arc lengths become large. The simplest way to decrease $\Delta s$ is by reducing $\Delta x$, but that will increase the computational workload drastically and include a large number of redundant or unnecessary surface points. Instead, extra points are inserted between points in the height profile when $\Delta s>\lambda / 4$ (see, e.g., Ref. 26). The extra points are found by cubic spline interpolations and, consequently, $\Delta x$ is no longer constant and Eq. (14) yields

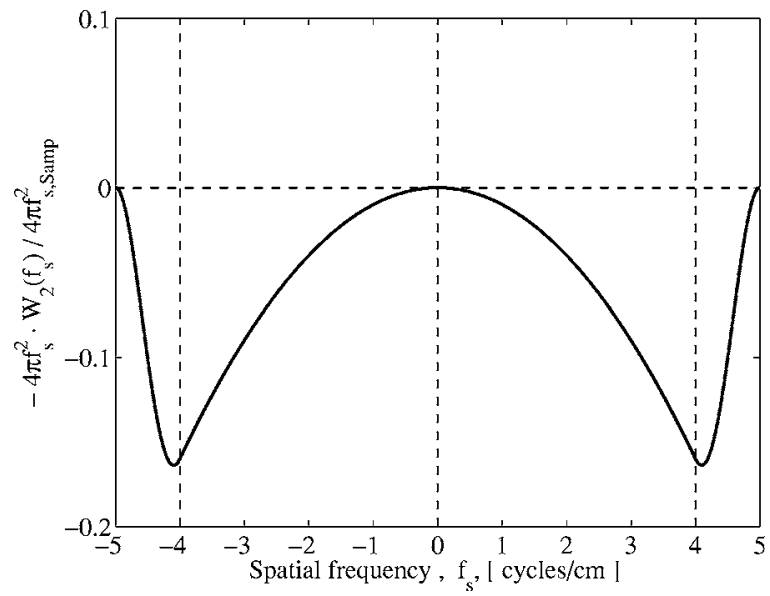

FIG. 3. Frequency domain window applied to obtain the surface second order derivative, $d^{2} h / d x^{2}$. 


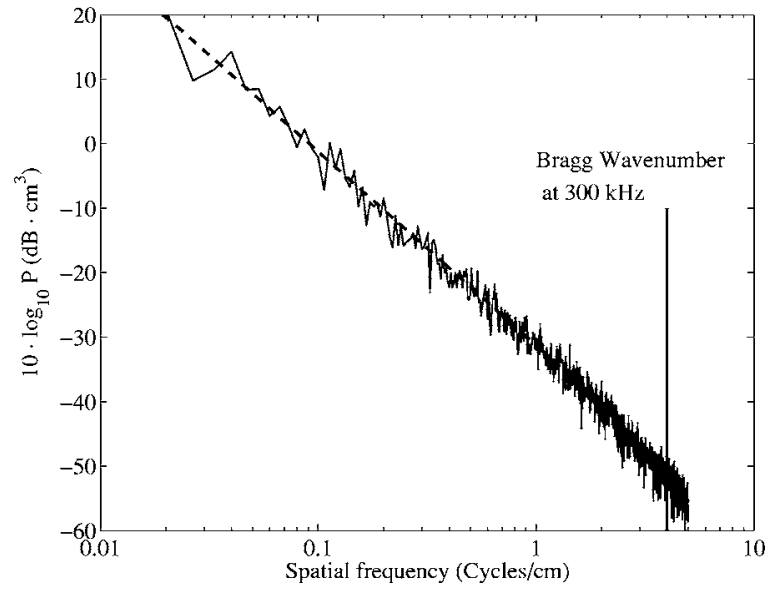

FIG. 4. Power spectrum of the interface roughness between the water and sediment; the spectrum has been acquired by optical means, i.e., stereophotogrammetric equipment. The dotted line represents the two-power law power spectrum according to Eq. (4) with the parameters listed in Table I. The solid, noisy, line is an average of five realizations.

$$
\Delta s\left(x_{n}\right)=\gamma\left(x_{n}\right) \Delta x\left(x_{n}\right) .
$$

The number of extra points required depends inversely on minimum spatial frequency. If $f_{s}^{(\mathrm{min})}$ decreases, the height profile will contain larger height values and arc lengths will increase. However, the minimum spatial frequency applied here only results in a few percent extra surface points. Equation (16) is applied in the implementation described in Sec. III.

Figure 4 shows the roughness power spectrum of the modeled interface; the spectrum shows an average of 5 roughness power spectra, and the maximum Braggwavenumber for backscattering at $300 \mathrm{kHz}(=4$ cycles $/ \mathrm{cm})$ is indicated by the solid vertical line.

\section{B. Sediment density variations}

The seabed may typically consist of sand, mud, stones, plants, and different animal species, but in this work only a sandy seabed sediment is considered. Experiments of the vertical and horizontal density variations have been carried out at the SAX99, and this section is solely based on results obtained by Tang et al. ${ }^{20}$ The experiments showed that the density variations are strongest within the first $5 \mathrm{~mm}$ of the sediment, that is, the transition layer or unconsolidated sediment. For frequencies below $100 \mathrm{kHz}$, i.e., wavelengths above approximately $1.5 \mathrm{~cm}$, the density inhomogeneities do not contribute to the scattering as the wavelength is greater than the thickness of the transition layer. At frequencies above $100 \mathrm{kHz}$ inhomogeneities are believed to affect scattering significantly.

The 3D spatial variations in the upper sediment layer, that is, 0 to $6 \mathrm{~cm}$, have been measured with an in situ measurement of porosity (IMP) system that measures the variability of the electrical conductivity within the sediment. The vertical density variations are considered in the following; Eqs. (17)-(21) are taken from Ref. 20 [Eqs. (11)-(15)]. For a sandy sediment the mean density as a function of depth is

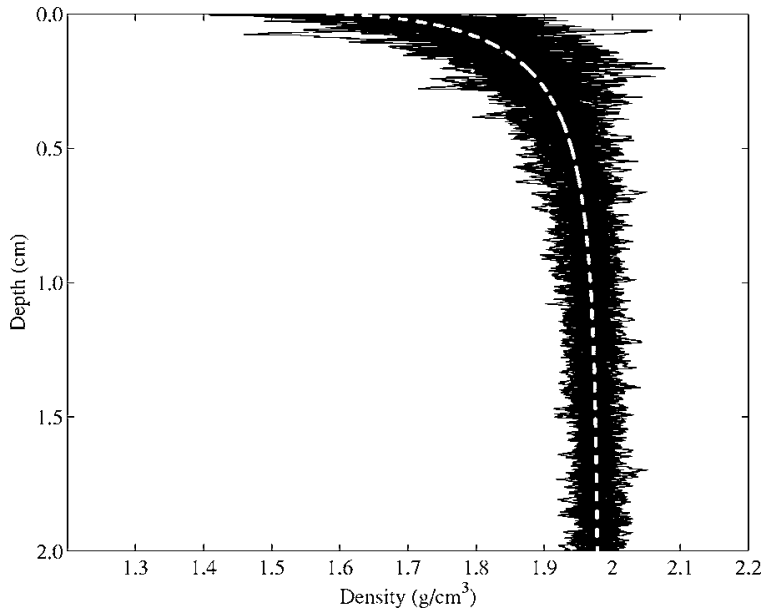

FIG. 5. A total of 32 synthetic density profiles based on density parameters listed in Table II and adopted from Ref. 20. The white dotted line represents the mean density profile, $\rho_{m}(z)$, given in Eq. (17).

$$
\rho_{m}(z)=1.98-0.4 e^{-3.5 z^{0.6}}
$$

where $\rho$ is measured in $\mathrm{g} / \mathrm{cm}^{3}$ and the depth $z$ in $\mathrm{cm}$. The relative density variability,

$$
\epsilon=\frac{\rho(x, y, z)-\rho_{m}(z)}{\rho_{m}(z)},
$$

is spatially nonstationary and follows the trend

$$
\epsilon_{m}=0.0152-0.096 e^{-3.7 z^{0.82}} \text {. }
$$

The relative density variation is normalized by its trend profile, Eq. (19),

$$
\eta=\frac{\epsilon(x, y, z)}{\epsilon_{m}(z)},
$$

where $\eta$ is assumed to be a spatial stationary process. In the vertical direction the power law spectrum yields

$$
P_{z}\left(f_{s}\right)=\frac{w}{f_{s}^{\gamma_{\rho}}},
$$

where $w=6.76 \times 10^{-2} \mathrm{~cm}^{1-\gamma_{\rho}}$ and $\gamma_{\rho}=2.17$. The power law has been verified up to a spatial frequency of approximately $f_{s}=20$ cycles $/ \mathrm{cm}$, which corresponds to a spatial resolution of $0.5 \mathrm{~mm}$ [see Ref. 20 (Fig. 14)]; above this frequency the noise floor of the IMP is reached. From these relations stochastic realizations of the sediment density are generated as a function of depth (see Fig. 5).

\section{The equivalent roughness profile}

The high density variations in the first millimeters below the water-sediment interface, i.e., the unconsolidated sediment, are believed to contribute significantly to the backscattered field. In order to include density variations in a model that only accounts for interface roughness scattering, an equivalent roughness approach is presented here. Considered from an acoustic point of view, there are numerous interfaces, or spatially distributed impedance contrasts, within the first $5 \mathrm{~mm}$ that contribute to the wave interaction. Here, the interface is redefined in terms of the density variations: For 
TABLE II. Applied sediment density parameters.

\begin{tabular}{lll}
\hline \hline Direction & Type & Parameters \\
\hline Vertical & Power law & $\begin{array}{l}\gamma_{\rho}=2.17 \\
w=0.0676 \mathrm{~cm}^{1-\gamma_{\rho}} \\
\end{array}$ \\
& & $\left(f_{s}=0.3-30 \mathrm{~cm}^{-1}\right)$ \\
Horzontal & $\operatorname{AR}(1)$ & $\kappa=0.45$ \\
\hline \hline
\end{tabular}

each point along the height profile a Monte Carlo realization like the ones shown in Fig. 5 is carried out, and the depth where the maximum density is found, $z\left(x_{n}, \rho_{\max }\right)$, forms the new height profile value. At the present stage the equivalent roughness is defined as

$$
h_{\mathrm{eq}}\left(x_{n}\right)=h\left(x_{n}\right)-z\left(x_{n}, \rho_{\max }\right) .
$$

Along the horizontal direction the density variations also follow a power spectrum [see Ref. 20 (Fig. 15)], but experimental data are only provided in the frequency range 0.06 to 2 cycles $/ \mathrm{cm}$, and at a minimum depth of $1 \mathrm{~cm}$. Here, we are interested in wave numbers up to 4 cycles $/ \mathrm{cm}$ at sediment depths from 0 to $1 \mathrm{~cm}$. Because $z\left(x_{n}, \rho_{\max }\right)$ tends to be white noise along the horizontal direction, $h_{\mathrm{eq}}\left(x_{n}\right)$ is filtered with an $\operatorname{AR}(1)$ low-pass filter (see, e.g., Ref. 27) with coefficient $\kappa$, that is,

$$
h_{\text {eqft }}\left(x_{n}\right)=\kappa h_{\text {eqft }}\left(x_{n-1}\right)+(\kappa-1) h_{\text {eq }}\left(x_{n}\right) .
$$

A single part of the results presented in Sec. IV is given in advance by anticipating that $\kappa=0.45$; this value gives an equivalent roughness profile, $h_{\text {eqft }}\left(x_{n}\right)$, that results in modeled backscattering strengths that approach experimental data. The parameters applied for the generation of equivalent roughness profiles are listed in Table II.

Figure 6 shows the spatial power spectra of the equivalent roughness profile together with the equivalent roughness interface in the case where the interface acquired by optical means is perfectly flat, that is, $h\left(x_{n}\right) \equiv x_{0}$. The optically acquired two-power law, i.e., Eq. (4), is also shown. The power

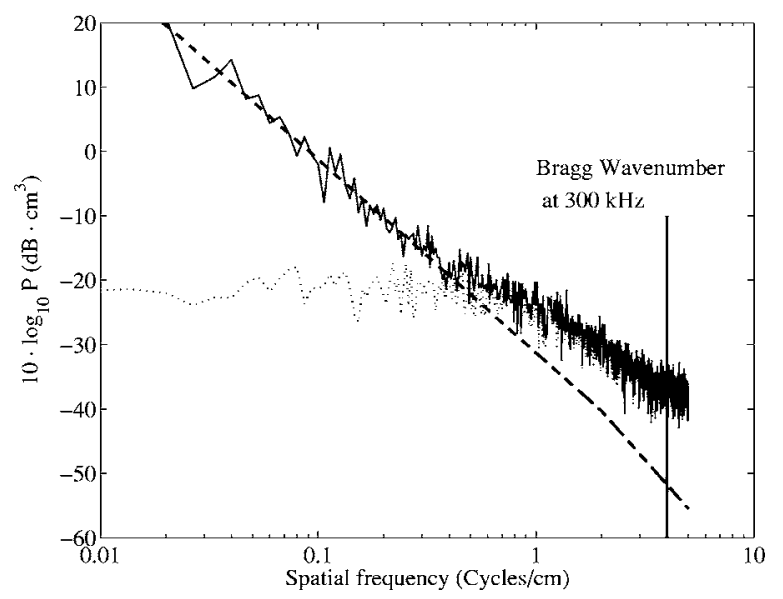

FIG. 6. Spatial power spectra obtained as an average of five equivalent roughness realizations based on spatial density parameters given in Table II. The solid line (The thin dotted line (:) is the roughness solely estimated from the density variations. The dashed line (- - ) is the two-power law, see Eq. (4), based on parameters listed in Table I.

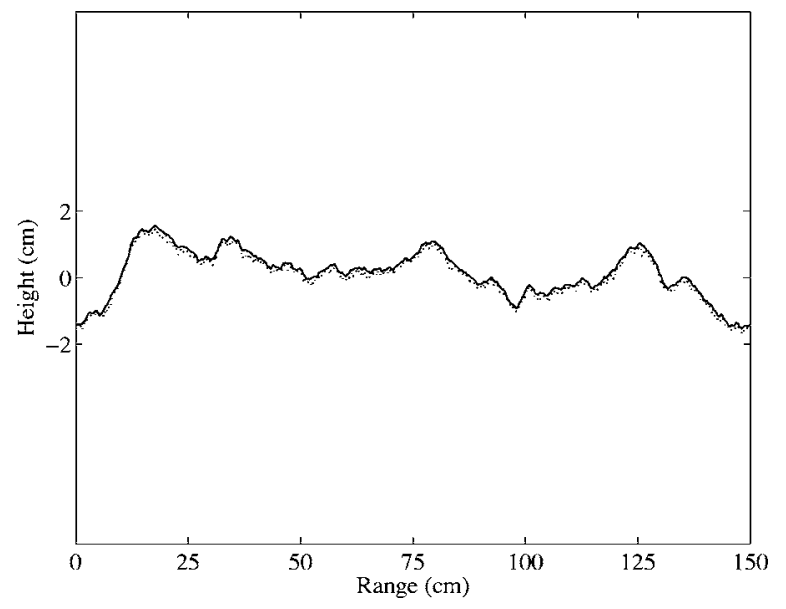

FIG. 7. Stochastic realizations of height profiles. The solid line (represents the interface roughness and the dotted line $(:)$ the equivalent roughness.

spectra of the equivalent roughness profiles exhibit higher levels for large wave numbers, i.e., from 0.4 to 4 cycles $/ \mathrm{cm}$, than the optically acquired two-power law. Hence, an increase in the backscattering level is expected there.

Figure 7 is an example of a 1.5-m-long height profile based on optical data only, together with the corresponding equivalent roughness profile. Figure 8 is Fig. 7 zoomed to the range between 10 and $30 \mathrm{~cm}$.

In the next section the field equations and the method applied to calculate the scattered field from the interface are presented.

\section{FIELD EQUATIONS}

In this work wave field propagation and scattering is considered in two dimensions, a consequence of the heightfield to height-profiles approximation presented in beginning of Sec. II. The $e^{-i \omega t}$ time dependence is assumed. Hence, a two-dimensional diverging outgoing wave is described in terms of the Hankel function of the first kind, $H_{0}^{(1)}(z)$. A plane wave incident on the water-sediment interface on the

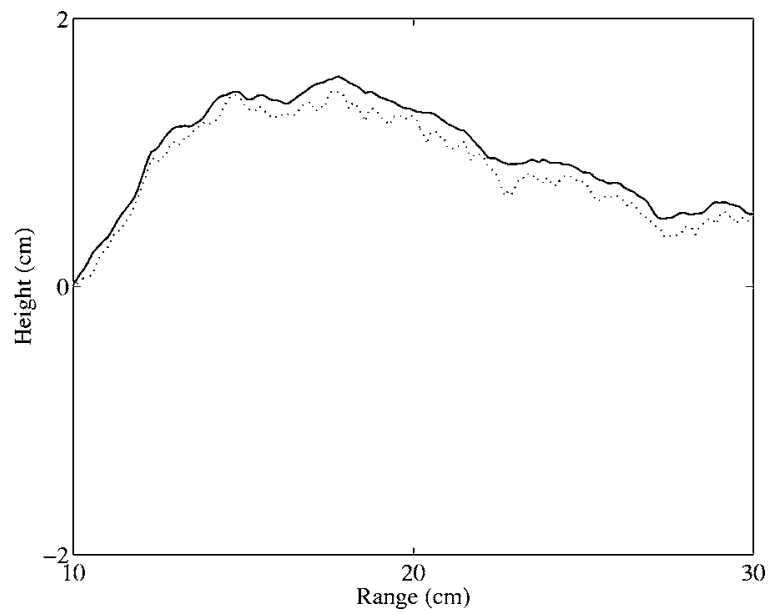

FIG. 8. The height profile realization from Fig. 7 zoomed. The solid line -) represents the interface roughness and the dotted line (:) the equivalent roughness. Note, the equivalent roughness exhibits higher small scale variations. 


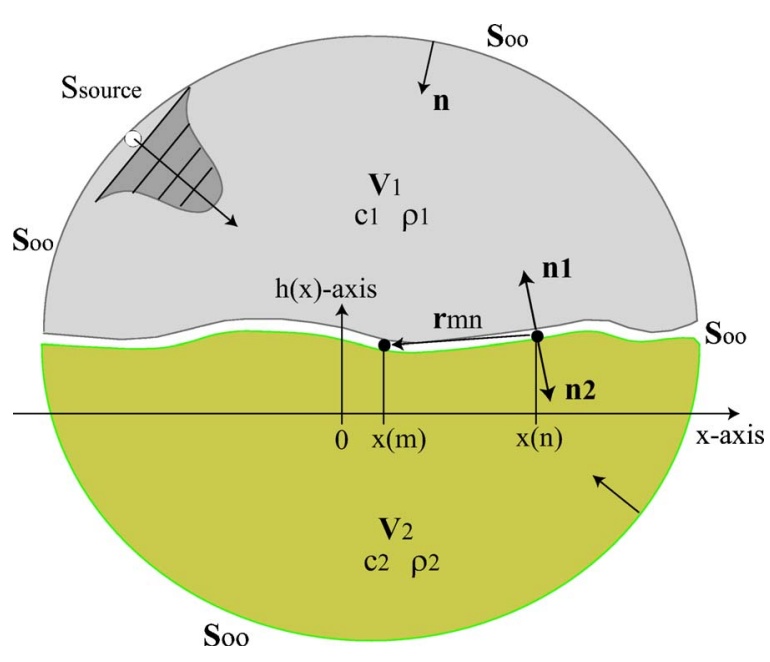

FIG. 9. The geometry applied for the fluid-fluid model represented by the coupled integral equations, Eqs. (24)-(26). In the water, i.e., medium 1, a tapered plane wave is incident from the infinity. Medium 2 represents the homogeneous sediment.

seabed is considered. The sediment is modeled as a fluid, i.e., the acoustic properties are fully described in terms of the mass density and sound speed. The backscattered pressure from the interface is the subject here. In Fig. $9 V_{1}$ represents the water medium, with density $\rho_{1}$ and sound speed $c_{1}$, and $V_{2}$ represents the sediment with density $\rho_{2}$ and sound speed $c_{2}$. Within $V_{1}$ a source at infinity generates a plane wave incident on the interface between the water and sediment; the plane wave is tapered, i.e., it is of finite extent and excites only a limited part of the seabed.

The field on the surface, $S_{\infty}$, at infinity obeys Sommerfeld's radiation condition, i.e., the field vanishes here. The pressure $p$ at an observation point $\mathbf{r}$ within or on the boundary of $V_{1}$ can be expressed in terms of the Kirchhoff Helmholtz integral equation (see, e.g., Ref. 28):

$$
\begin{aligned}
\alpha_{1} p(\mathbf{r})= & p^{i}(\mathbf{r})+\frac{1}{4 i} \int_{-\infty}^{\infty}\left(p\left(\mathbf{r}^{\prime}\right) \frac{\partial H_{0}^{(1)}\left(k_{1}\left|\mathbf{r}-\mathbf{r}^{\prime}\right|\right)}{\partial n^{\prime}}\right. \\
& \left.-H_{0}^{(1)}\left(k_{1}\left|\mathbf{r}-\mathbf{r}^{\prime}\right|\right) \frac{\partial p\left(\mathbf{r}^{\prime}\right)}{\partial n^{\prime}}\right) d s^{\prime}
\end{aligned}
$$

where the integral represents the field scattered from the interface $s^{\prime}, p^{i}$ is the incoming field, $\mathbf{r}^{\prime}$ is a point on $s^{\prime}$ and serves as an integration variable, $k_{1}=\omega / c_{1}$ is the acoustic wave number in the water, and $\partial / \partial n^{\prime}=\nabla \cdot \hat{\mathbf{n}}^{\prime}$ is the gradient projected onto the surface normal vector $\hat{\mathbf{n}}^{\prime}$ of unit length on $s^{\prime}$. Finally, the constant $\alpha_{1}$ on the left hand side of Eq. (24) is, for $j=1$, given by

$$
\alpha_{j}= \begin{cases}1, & \text { if } \mathbf{r} \text { is inside } V_{j}, \\ \frac{1}{2}, & \text { if } \mathbf{r} \text { is on the boundary of } V_{j}, \\ 0, & \text { if } \mathbf{r} \text { is outside } V_{j} .\end{cases}
$$

In the sediment, i.e., in $V_{2}$, the integral equation becomes

$$
\begin{aligned}
-\alpha_{2} p(\mathbf{r})= & \frac{1}{4 i} \int_{-\infty}^{\infty}\left(p\left(\mathbf{r}^{\prime}\right) \frac{\partial H_{0}^{(1)}\left(k_{2}\left|\mathbf{r}-\mathbf{r}^{\prime}\right|\right)}{\partial n^{\prime}}\right. \\
& \left.-H_{0}^{(1)}\left(k_{2}\left|\mathbf{r}-\mathbf{r}^{\prime}\right|\right) \frac{\partial p\left(\mathbf{r}^{\prime}\right)}{\partial n^{\prime}}\right) d s^{\prime},
\end{aligned}
$$

where there is no incoming field from within $V_{2}, \alpha_{2}$ is defined in Eq. (25) with $j=2$, and the sign of the integral has been reversed due to a $180^{\circ}$ reversal of the normal vector, $\hat{\mathbf{n}}^{\prime}$ (see Fig. 9). The two integral equations, Eqs. (24) and (26), are coupled through the boundary conditions at the watersediment interface as

$$
\begin{aligned}
& p_{1}\left(\mathbf{r}^{\prime}\right)=p_{2}\left(\mathbf{r}^{\prime}\right), \\
& \frac{\partial p_{1}\left(\mathbf{r}^{\prime}\right)}{\partial n^{\prime}}=\frac{1}{\mu} \frac{\partial p_{2}\left(\mathbf{r}^{\prime}\right)}{\partial n^{\prime}},
\end{aligned}
$$

where $\mu=\rho_{2} / \rho_{1}$.

The gradient of the Hankel function projected onto the surface normal [the left term inside the integral of Eq. (24) and Eq. (26)] is considered next. The surface derivative of the zeroth-order Hankel function of the first kind projected onto the surface normal is

$$
\frac{H_{0}^{(1)}\left(k\left|\mathbf{r}-\mathbf{r}^{\prime}\right|\right)}{\partial n^{\prime}}=k H_{1}^{(1)}\left(k\left|\mathbf{r}-\mathbf{r}^{\prime}\right|\right)\left(\hat{\mathbf{r}}^{\prime} \cdot \hat{\mathbf{n}}^{\prime}\right),
$$

where $\hat{\mathbf{r}}^{\prime}=\left(\mathbf{r}-\mathbf{r}^{\prime}\right) /\left|\mathbf{r}-\mathbf{r}^{\prime}\right|$.

In the following, the position variables are written in terms of a discrete surface and, thus, the observation point vector $\mathbf{r}$ is given by $\left(x_{m}, h\left(x_{m}\right)\right)$, where $m=1,2, \ldots, N$; the integration variable $\mathbf{r}^{\prime}$ is given by $\left(x_{n}, h\left(x_{n}\right)\right)$, where $n$ $=1,2, \ldots, N$. The distance between the observation point $m$ and integration point $n$ is

$$
r_{m n}=\sqrt{\left(x_{m}-x_{n}\right)^{2}+\left[h\left(x_{m}\right)-h\left(x_{n}\right)\right]^{2}},
$$

and the unit vector pointing from $n$ to $m$ is

$$
\hat{\mathbf{r}}_{m n}=\frac{1}{r_{m n}}\left[\begin{array}{c}
x_{m}-x_{n} \\
h\left(x_{m}\right)-h\left(x_{n}\right)
\end{array}\right] \text {. }
$$

The unit surface normal vector is given by

$$
\hat{\mathbf{n}}\left(x_{n}\right)=\frac{1}{\gamma\left(x_{n}\right)}\left[\begin{array}{c}
-\left.(d h / d x)\right|_{x=x_{n}} \\
1
\end{array}\right],
$$

where $\gamma\left(x_{n}\right)$ is defined in Eq. (15). The $n$th line segment that points towards the $m$ th observation point has an effective length of

$$
\boldsymbol{\kappa}_{m n}=\Delta s(n) \hat{\mathbf{n}}\left(x_{n}\right) \cdot \hat{\mathbf{r}}_{m n},
$$

and combining Eqs. (14), (31), and (32) in Eq. (33) yields

$$
\kappa_{m n}=\Delta x(n) \frac{-\left.\left(x_{m}-x_{n}\right)(d h / d x)\right|_{x=x_{n}}+\left[h\left(x_{m}\right)-h\left(x_{n}\right)\right]}{r_{m n}},
$$

a factor applied in the discretization of the first integrand in Eq. (26). In order to find the scattered pressure the pressure and pressure gradient on rough interface must be determined. The establishment of the matrix equations for the coupled 
problem follows Chan, ${ }^{11}$ who used the collocation method to obtain the following set of equations,

$$
\begin{aligned}
& p^{(\mathrm{inc})}\left(x_{m}\right)=\sum_{n=1}^{N} a_{m n} F_{1}\left(x_{n}\right)+\sum_{n=1}^{N} b_{m n} F_{2}\left(x_{n}\right), \\
& 0=\sum_{n=1}^{N} c_{m n} F_{1}\left(x_{n}\right)+\sum_{n=1}^{N} d_{m n} F_{2}\left(x_{n}\right),
\end{aligned}
$$

where $F_{1}(x)=p(x)$ and $F_{2}(x)=\gamma\left(x_{n}\right) \partial p(x) / \partial n$. For $m \neq n$,

$$
\begin{aligned}
& a_{m n}=-\frac{i k_{1}}{4} \kappa_{m n} H_{1}^{(1)}\left(k_{1} r_{m n}\right), \\
& b_{m n}=\Delta x(n) \frac{i}{4} H_{0}^{(1)}\left(k_{1} r_{m n}\right), \\
& c_{m n}=\frac{i k_{1}}{4} \kappa_{m n} H_{1}^{(1)}\left(k_{2} r_{m n}\right), \\
& d_{m n}=-\mu \Delta x(n) \frac{i}{4} H_{0}^{(1)}\left(k_{2} r_{m n}\right),
\end{aligned}
$$

where $\kappa_{m n}$ is defined by Eq. (34). For $m=n$ the coefficients become

$$
\begin{aligned}
& a_{m m}=\frac{1}{2}-\frac{h^{\prime \prime}(x) \Delta x(m)}{4 \pi \gamma_{m}^{2}}, \\
& b_{m m}=\Delta x \frac{i}{4} H_{0}^{(1)}\left[k_{1} \Delta x(m) \gamma_{m} /(2 e)\right], \\
& c_{m m}=\frac{1}{2}+\frac{h^{\prime \prime}(x) \Delta x(m)}{4 \pi \gamma_{m}^{2}}, \\
& d_{m m}=-\mu \Delta x(m) \frac{i}{4} H_{0}^{(1)}\left[k_{1} \Delta x(m) \gamma_{m} /(2 e)\right] .
\end{aligned}
$$

In matrix form the following is obtained,

$$
\left[\begin{array}{cccccc}
a_{11} & b_{11} & a_{12} & \ldots & a_{1 N} & b_{1 N} \\
c_{11} & d_{11} & c_{12} & \ldots & c_{1 N} & d_{1 N} \\
\vdots & \vdots & \ddots & \ddots & b_{2 N} & b_{2 N} \\
\vdots & \vdots & \ldots & \ldots & \ldots & \ldots \\
a_{N 1} & b_{N 1} & a_{N 2} & \ldots & a_{N N} & b_{N N} \\
c_{N 1} & d_{N 1} & c_{N 2} & \ldots & c_{N N} & d_{N N}
\end{array}\right]\left[\begin{array}{c}
F_{1}\left(x_{1}\right) \\
F_{2}\left(x_{1}\right) \\
\vdots \\
\vdots \\
F_{1}\left(x_{N}\right) \\
F_{2}\left(x_{N}\right)
\end{array}\right]=\left[\begin{array}{c}
p^{i}\left(x_{1}\right) \\
0 \\
\vdots \\
\vdots \\
p^{i}\left(x_{N}\right) \\
0
\end{array}\right] .
$$

When the pressure and the pressure gradient on the interface have been determined the resulting field at any observation point in the water column can be determined. The far field expression for the scattered field is

$$
\begin{aligned}
p_{s c}(\mathbf{r})= & \frac{1}{4 i} \sum_{n=1}^{N} \sqrt{\frac{2}{\pi k_{1} r_{n}}} e^{i k r_{n}} e^{-i \pi / 4}\left[i p\left(x_{n}\right)-\frac{\partial p}{\partial n}\left(x_{n}\right)\right] \\
& \times \Delta x(n),
\end{aligned}
$$

where $r_{n}=\left|\mathbf{r}-\mathbf{r}_{n}\right|$.

\section{APPROXIMATIVE SOLUTION FOR LARGE SURFACES}

For very long height profiles the matrix in Eq. (45) becomes extremely large since a sonar's field of view may cover several square meters. Suppose a height profile of length $20 \mathrm{~m}$ is required for modeling the sonar beam; a scattering computation of a $300-\mathrm{kHz}$ wave requires a height field resolution equal to $1 \mathrm{~mm}$, which yields a matrix of size $20000^{2}$ or 2.3 Gigabytes. Iterative solutions to Eq. (45) can be applied such as the conjugant gradient method (see Ref. 11) or the forward-backward method, similar to the GaussSeidel procedure (see Ref. 10). Here the direct method is maintained, but the matrix is reduced to a sparse band matrix by the insertion of zeros outside a certain number of diagonals,

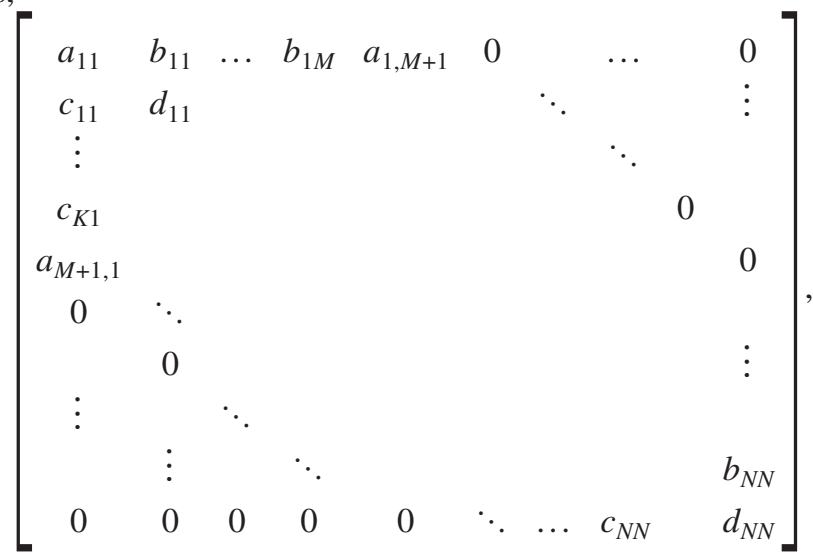

with $M$ diagonals below and $M$ diagonals above the main diagonal. This choice is based on the fact that the Hankel function decays with range or, expressed in physical terms, the pressure at a given observation point on the surface mainly depends on the nearest neighbor points due to the geometrical spreading of the scattered waves.

\section{RESULTS}

The incoming field is formed as a tapered plane wave according to Ref. 9 [Eq. (11)], where the tapering parameter, $g$, of the incoming plane wave is equal to $L / 4$, and $L$ is the height profile length. Results are presented in terms of the scattering strength,

$$
\mathrm{SS}=10 \log _{10} \sigma\left(\theta, \theta_{s}\right),
$$

where $\sigma\left(\theta, \theta_{s}\right)$ is the dimensionless scattering cross section. For 2D wave propagation it is given by [see, e.g., Ref. 9, Eq. (13)]

$$
\sigma\left(\theta, \theta_{s}\right)=\left\langle I_{s}\left(\theta_{s}\right)\right\rangle r / I_{\mathrm{inc}} L,
$$

where $I_{\text {inc }}$ is the incident intensity and $\left\langle I_{s}\left(\theta_{s}\right)\right\rangle$ is the scattered intensity in the far field range $r$ and averaged over numerous surface realizations in the direction $\theta_{s}$. Finally, $L$ is the profile length. Thus, $\sigma$ is the ratio of the acoustic power scattered in direction $\theta_{s}$ to the power of the incoming field with grazing angle $\theta$, and, hence, it is comparable with the scattering cross section applied for 3D scattering problems.

Wendelboe, Jacobsen, and Bell: An equivalent roughness model 


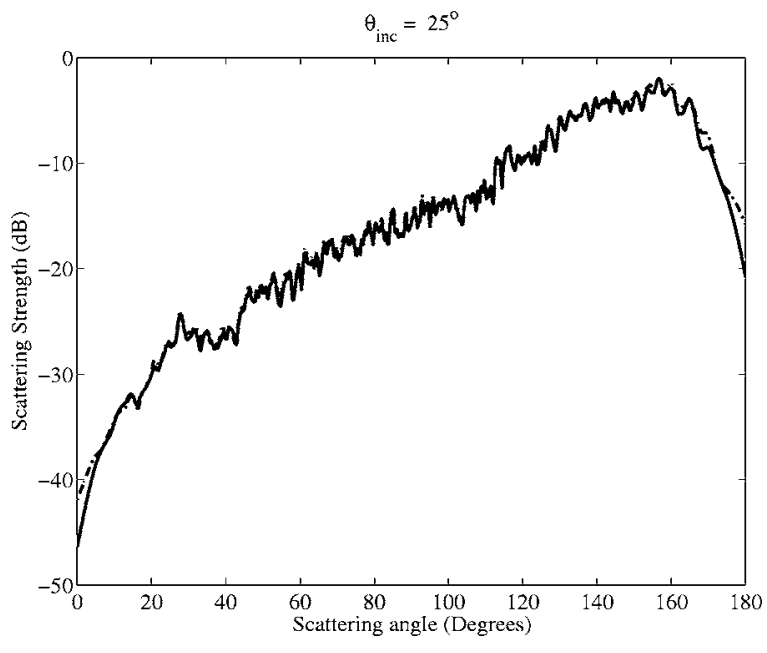

FIG. 10. Bistatic scattering strength for the equivalent roughness surface subject to a pressure release boundary condition. The incoming wave has a grazing angle of $25^{\circ}$. The solid line (- $)$ is the full matrix solution and the dash-dot line (---) is the band-matrix solution with $M=80$

The equivalent roughness profiles applied in the following correspond to the type of realizations shown in Figs. 7 and 8; the seabed parameters are depicted in Tables I and II. The AR(1)-parameter value, $\kappa=0.45$ [see Eq. (23)] is found to be an acceptable choice since the modeled backscattering strengths match the experimental data reasonably well (see below). The spatial ground range resolution of a height profile is $\lambda / 5=1 \mathrm{~mm}$, and the acoustic parameters used in the simulations are, unless anything else is specified, as follows: the speed of sound in water $c_{1}=1500 \mathrm{~m} / \mathrm{s}$, the sound speed ratio $\delta=c_{2} / c_{1}=1.165$, the water density $\rho_{1}=1 \mathrm{~g} / \mathrm{cm}^{3}$, and the homogeneous sediment density $\rho_{1}=1.8 \mathrm{~g} / \mathrm{cm}^{3}$.

Figure 10 shows the bistatic scattering strength for an incident field with a grazing angle, $\theta_{i}=25^{\circ}$, and a height profile length of $120 \lambda$. The interface has a pressure-release boundary condition, which is established by setting $\rho_{2}$ $=10^{-5} \mathrm{~g} / \mathrm{cm}^{3}$. For the scattering problem on a pressure release surface the Kirchoff-Helmholtz integral equation reduces to

$$
p^{i}(\mathbf{r})=\frac{1}{4 i} \int_{-\infty}^{\infty} H_{0}^{(1)}\left(k_{1}\left|\mathbf{r}-\mathbf{r}^{\prime}\right|\right) \frac{\partial p\left(\mathbf{r}^{\prime}\right)}{\partial n^{\prime}} d s^{\prime},
$$

and the solution, given by Eqs. (4), (5), and (8) in Ref. 9, is applied as a first validation of the model presented here.

In Fig. 10 the full matrix solution based on Thorsos's solution is shown together with the solution provided by the band-matrix method, with $M=80$, where $M$ is the number of nonzero diagonals to each side of the main diagonal. For the scattering angles in the range from $0^{\circ}$ to $10^{\circ}$ the error decreases from $5 \mathrm{~dB}$ down to approximately $1 \mathrm{~dB}$. From $10^{\circ}$ up to approximately $160^{\circ}$ the solutions continue to coincide within $1 \mathrm{~dB}$.

Figure 11 shows the backscattering error between the full matrix solution and the band matrix solution for different values of $M$, i.e., $M=20,40,80,160,320$. The grazing angles under consideration are $\theta=1^{\circ}, 5^{\circ}, 10^{\circ}, 20^{\circ}, 30^{\circ}, \ldots, 90^{\circ}$, and each point is an average of 20 surface realizations of length $200 \lambda=1 \mathrm{~m}$. The errors increase dramatically for grazing

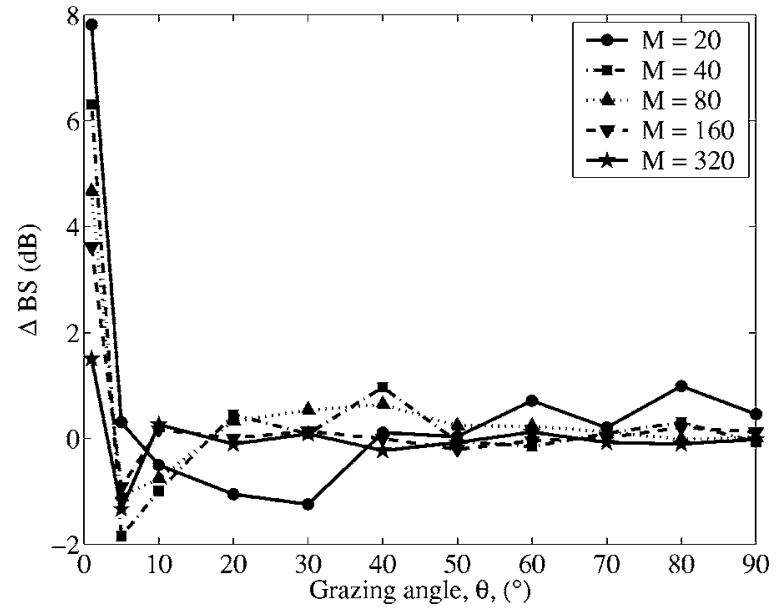

FIG. 11. Backscattering difference in $\mathrm{dB}$ between the full matrix solution and the band matrix solution for different values of $M$ as a function of grazing angle. The curves are averages of 20 surface realizations of length $200 \lambda$.

angles below $5^{\circ}$, where the most sparse matrix solution, i.e., $M=20$, has a maximum error of approximately $8 \mathrm{~dB}$ at $\theta$ $=1^{\circ}$. The smallest error, at $\theta=1^{\circ}$, is $1.8 \mathrm{~dB}$ for $M=320$. For grazing angles above $5^{\circ}$ the error for $M=20$ is within $1.5 \mathrm{~dB}$, $1 \mathrm{~dB}$ for $M=40$, and for higher values of $M$ below $0.5 \mathrm{~dB}$. Apart from computational accuracy, the computational efficiency is another aspect that must be taken into account: The capability of estimating the backscattered pressure from long height profiles is needed. Therefore, a band matrix with $M$ $=20$ is chosen. It is unlikely that a sonar is directed toward the seabed with a grazing angle of less than $5^{\circ}$ and, therefore, with $M=20$, an expected error of $1.5 \mathrm{~dB}$ is considered to be acceptable.

The required $\mathrm{CPU}$ time as a function of height profile length has been invested on a $3 \mathrm{GHz}$ Pentium4 ${ }^{\mathrm{TM}}$ processor with $512 \mathrm{MB}$ RAM, and computations have been made in Matlab $^{\mathrm{TM}}$ on a Windows-XPTM operating system. The maximum profile length that can be computed on the current platform is $N=25000$, which takes approximately $95 \mathrm{~s}$; larger matrices result in lack of workspace memory and cannot be carried out. The applied incremental ground range resolution is $1 \mathrm{~mm}$, which yields a profile length of $25 \mathrm{~m}$. The interpolation that is carried out when the distances between adjacent points on the height profile are larger that $\lambda / 4$ typically increases the number of elements, $N$, by $3 \%$, thus the actual matrix length is $N=25750$. Computation of a matrix of length $N=15000$, i.e., a profile length of $15 \mathrm{~m}$, takes approximately $40 \mathrm{~s}$, and a matrix with $N=5000$, i.e., a profile length of $5 \mathrm{~m}$, takes approximately $15 \mathrm{~s}$.

Figure 12 shows different backscattering strengths computed from equivalent roughness profiles; the grazing angle resolution is $0.5^{\circ}$, and each curve represents an average of 50 surface realizations. All band matrix solutions use $M=20$, i.e., 20 nonzero diagonals below and above the main diagonal of Eq. (47). The backscattering strengths from the equivalent roughness profiles of length $L_{x}=200 \lambda$, i.e., $1 \mathrm{~m}$, have been computed by the full matrix solution and the band matrix solution. Additionally, backscattering strengths from equivalent roughness profiles of length $L_{x}=2000 \lambda$, i.e., 


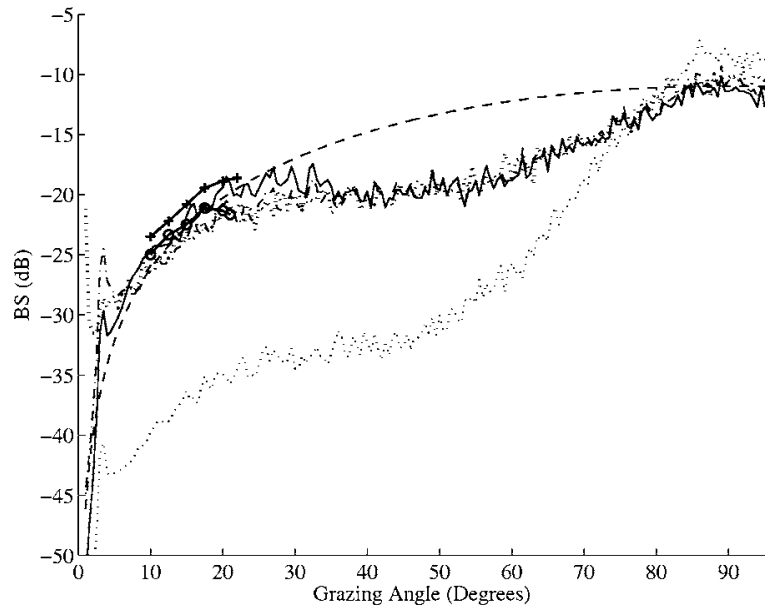

FIG. 12. Backscattering strength as a function of $\theta$ with $\Delta \theta=0.5^{\circ}$. All band matrix solutions (BMS) use $M=20$. The solid line ( $\longrightarrow$ ) is the full matrix solution, the dash-dot line (---) is the BMS, and both curves are based on the equivalent roughness profile (ERP). The thin dotted line (:) is the BMS from a height profile only based on stereo photogrammetry. The ERPs explained so far have a length of $200 \lambda$. The thick dotted line (:) is BMS, with an ERP length of $2000 \lambda$; the cross and circle marked lines, i.e., $(+)$, and $(-)$, represent the SAX99 data; finally, the thin dashed line $(-)$ is Lamberts law.

$10 \mathrm{~m}$, have been computed with the band matrix solution. The three solutions coincide within a few decibels with the SAX99 data, i.e., XBAMS and BAMS presented in Ref. 21 (Fig. 5, p. 10) for grazing angles between $10^{\circ}$ and $22^{\circ}$. The backscattering strengths calculated from the interface found by optical means, i.e., the stereo-photogrammetric equipment, are for $\theta=5^{\circ}$ to $50^{\circ}$, approximately $15 \mathrm{~dB}$ weaker than the strengths found from the equivalent roughness profiles. For grazing angles between approximately $50^{\circ}$ up to normal incidence, i.e., $90^{\circ}$, this difference decreases gradually. The model follows Lambert's law for small grazing angles as well as angles near normal incidence, i.e., for $\theta<30^{\circ}$ and $\theta>80^{\circ}$, respectively.

\section{DISCUSSION}

Apart from a strong spatial variability of the density, experiments have also revealed a high spatial variability of the sediment sound speed (see, for example, Refs. 20 and 25 ), but sound speed variability is not included in the equivalent roughness approximation.

The scattering model presented in Sec. III is adopted from radar theory, where the interface between air and ground has a significant impedance contrast, which consequently yields a weak wave penetration into the ground. Hence, interface scattering is the dominant scattering mechanism, and the air and ground can be considered as homogeneous media. Prior to the development of the equivalent roughness approach it was attempted to vary the sediment density along each discrete surface point; that is, the density ratio $\mu$, in Eqs. (40) and (44), was replaced by $\mu_{n}$, with $n$ $=1,2, \ldots, N$, where $N$ represents the total number of surface points. The same principle was applied by varying $k_{2}$, i.e., the sediment sound speed, along the height profile. However, simulations did not show any change in the shape of the backscattering curve, probably because the equations formulated in Sec. III are formulated strictly for interface roughness variations.

Another approach to compute the scattered field could be a finite element model, also for 1D height profiles, of the upper part of the sediment combined with a boundary value formulation for the interface. However, the method would probably become very computationally demanding and yet suffer from the lack of precise information regarding small scale density and sound speed variations in the upper part of the sediment.

It has also been attempted to apply the method presented by D. Kapp et al., ${ }^{10}$ but it yields more inaccurate results for the zeroth-order Born term than the band matrix approximation applied here, and it does not converge for higher order iterations.

It is important to emphasize that the optically acquired roughness parameters, shown in Table I, probably not are measured at the same date and time as the acoustic data. Except for the fact that BAMS is the correct site, it is not quite clear which set of parameters in Ref. 23 must be applied. However, it does not change the fact that the equivalent roughness approach lifts the backscattering level up to the levels obtained from experiments.

\section{CONCLUSION}

The equivalent roughness approximation yields, when $\kappa=0.45$, backscattering strengths at $300 \mathrm{kHz}$ that agree with experimental data acquired at SAX99. The band-matrix approximation, with $M=20$, yields backscattering errors of $8 \mathrm{~dB}$ for $\theta=1^{\circ}$; for grazing angles above $5^{\circ}$ backscattering errors are less that $1.5 \mathrm{~dB}$. Sonar simulations are not expected to be carried out for grazing angles of less than $5^{\circ}$, and, hence, the model is considered to have a sufficient accuracy for $M=20$. Computations have been carried out in Matlab6.5 ${ }^{\mathrm{TM}}$ on a PC with a Windows XPTM operating system, a $3 \mathrm{GHz}$ (Pentium4 ${ }^{\mathrm{TM}}$ ) processor, and $512 \mathrm{MB}$ RAM. The method allows computations of height profiles with 25000 elements that correspond to $25 \mathrm{~m}$ when the resolution is one-fifth of the wavelength and the wavelength is $\lambda$ $=0.5 \mathrm{~cm}$. The equivalent roughness approach combined with the band matrix method is well suited to model sandy seabed backscattering for artificially very high frequency sonar images.

\section{ACKNOWLEDGMENTS}

The authors are grateful to Dajun Tang at Applied Physics Laboratory, University of Washington, for answers related to the seabed model, and to Eric Thorsos, Applied Physics Laboratory, University of Washington, for general advice regarding the scattering problem. The authors also gratefully acknowledge advice from Per Christian Hansen, Informatics and Mathematical Modeling (IMM), Technical University of Denmark, regarding the band matrix solution. 


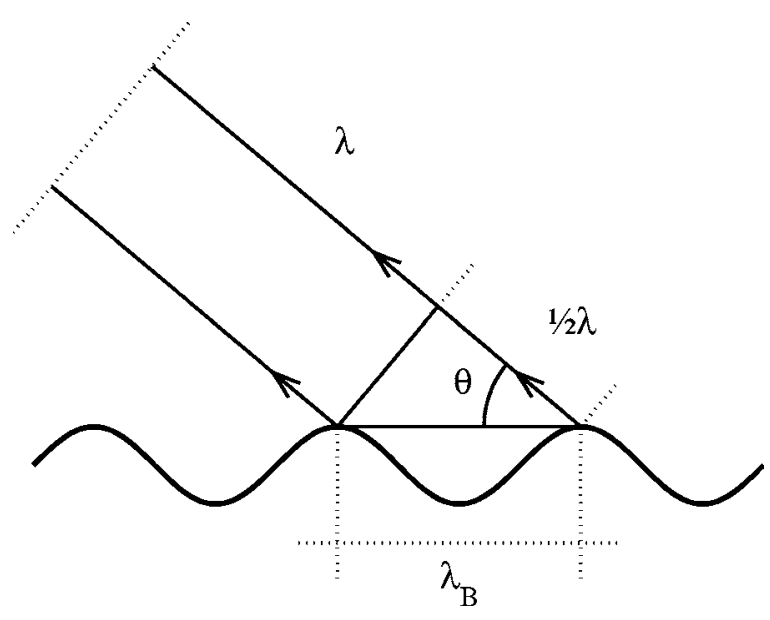

FIG. 13. Geometry applied to derive the Bragg wavelength.

\section{APPENDIX: THE BRAGG WAVELENGTH}

The Bragg wavelength, $\lambda_{B}$, for a rough interface is defined in terms of an incoming, monochromatic plane wave with wavelength, $\lambda$, and a grazing angle of incidence, $\theta$, that is,

$$
\lambda_{B}=\frac{\lambda}{2 \cos \theta}
$$

(see Fig. 13). Equation (A1) is equivalent to the reinforcement criterion for backscattering described by Urick. ${ }^{29}$ The Bragg frequency of the seabed roughness is

$$
f_{B}=\frac{2 \cos \theta}{\lambda} .
$$

At $300 \mathrm{kHz}$ the wavelength is $\lambda=0.5 \mathrm{~cm}$ when it is assumed that the sound speed is $c=1500 \mathrm{~m} / \mathrm{s}$; thus at zero grazing angle, i.e., $\theta=0$, one has $f_{B}=4 \mathrm{cycles} / \mathrm{cm}$, which corresponds to the vertical lines indicated in Figs. 1-3.

${ }^{1}$ E. Pouliquen, G. Canepa, L. Pautet, and A. P. Lyons, "Temporal variability of seafloor roughness and its impact on acoustic scattering," in Proceedings of the Seventh European Conference in Underwater Acoustics (ECUA2004), Delft, The Netherlands, 5-8 July 2004.

${ }^{2}$ J. A. Ogilvy, Theory of Wave Scattering from Random Rough Surfaces (IOP, London, 1991), Chap. 4.1.2.

${ }^{3}$ L. M. Brekhovskikh and Yu. P. Lysanov, Fundamentals of Ocean Acoustics. Third edition (AIP, Springer-Verlag, New York, 2003).

${ }^{4}$ E. I. Thorsos and D. R. Jackson, "Studies of scattering using numerical methods," Waves Random Media 3, S165-S190 (1991).

${ }^{5} \mathrm{~J}$. M. Bell, "Application of optical ray tracing techniques to the simulation of sonar images," Opt. Eng. (Bellingham) 36, 1806-1813 (1997).

${ }^{6}$ T. Capron, G. Hayward, and R. Chapmann, "A 3D simulator for the design and evaluation of sonar system instrumentation," Meas. Sci. Technol. 10, 1116-1126 (1999).

${ }^{7}$ E. I. Thorsos and S. L. Broschat, "An investigation of the small slope approximation for scattering from rough surfaces. Part I. Theory," J. Acoust. Soc. Am. 97, 2082-2093 (1995).
${ }^{8}$ S. L. Broschat and E. I. Thorsos, "An investigation of the small slope approximation for scattering from rough surfaces. Part II. Numerical studies," J. Acoust. Soc. Am. 101, 2615-2625 (1997).

${ }^{9}$ E. I. Thorsos, "The validity of the Kirchhoff approximation for rough surface scattering using a Gaussian roughness spectrum," J. Acoust. Soc. Am. 83, 78-92 (1988).

${ }^{10}$ D. A. Kapp and G. S. Brown, "A new numerical method for rough surface scattering calculations," IEEE Trans. Antennas Propag. 44, 711-721 (1996).

${ }^{11}$ C. H. Chan, L. Tsang, and Q. Li, "Monte Carlo simulations of large scale one-dimensional random rough surface scattering at near-grazing incidence: Penetrable case," IEEE Trans. Antennas Propag. 46, 142-149 (1998).

${ }^{12}$ D. R. Jackson, D. P. Winebrenner, and A. Ishmaru, "Application of the composite roughness model to high frequency bottom backscattering," J. Acoust. Soc. Am. 79, 1410-1422 (1986).

${ }^{13}$ D. R. Jackson and K. B. Briggs, "High frequency bottom backscattering: Roughness versus sediment volume scattering," J. Acoust. Soc. Am. 92, 962-977 (1992).

${ }^{14}$ P. D. Mourad and D. R. Jackson, "High-frequency sonar equation models for bottom backscatter and forward loss," in Proceedings of OCEANS'89 (1989), pp. 1168-1175.

${ }^{15}$ D. R. Jackson, "Models for scattering from the sea bed," Proc. IOA, Vol. 16, Part 6 (1994).

${ }^{16}$ K. L. Williams and D. R. Jackson, "Bistatic bottom scattering: Model, experiments, and model/data comparison," J. Acoust. Soc. Am. 103, 169181 (1998)

${ }^{17}$ S. Stanic, R. R. Goodman, K. B. Briggs, N. P. Chotiros, and E. T. Kennedy, "Shallow-water bottom reverberation measurements," IEEE J. Ocean. Eng. 23, 203-210 (1998).

${ }^{18}$ E. Pouligen and A. P. Lyons, "Backscattering from Bioturbated sediments at very high frequency," IEEE J. Ocean. Eng. 27, 388-402 (2002).

${ }^{19}$ K. L. Williams, D. R. Jackson, E. I. Thorsos, D. Tang, and K. B. Briggs, "Acoustic backscattering experiments in a well characterized sand sediment: Data/model comparisons using sediment fluid and Biot models," IEEE J. Ocean. Eng. 27, 376-387 (1998).

${ }^{20}$ D. Tang, K. B. Briggs, K. L. Williams, D. R. Jackson, E. I. Thorsos, and D. B. Percival, "Fine scale volume heterogeneity measurments in sand," IEEE J. Ocean. Eng. 27, 546-560 (2002).

${ }^{21}$ E. I. Thorsos, K. L. Williams, N. P. Chotiros, J. T. Christoff, K. W. Commander, C. F. Greenlaw, D. V. Holliday, D. R. Jackson, J. L. Lopes, D. E. McGehee, J. E. Piper, M. D. Richardson, and D. Tang, "An overview of SAX99: Acoustic measurements," IEEE J. Ocean. Eng. 26, 4-25 (2001).

${ }^{22} \mathrm{O}$. George and R. Bahl, "Simulation of backscattering of high frequency sound from complex objects and sand sea-bottom," IEEE J. Ocean. Eng. 20, 119-130 (1995).

${ }^{23}$ K. B. Briggs, D. Tang, and K. L. Williams, "Characterisation of interface roughness of rippled sand off Fort Walton Beach, Florida," IEEE J. Ocean. Eng. 27, 505-514 (2002).

${ }^{24} \mathrm{~K}$. B. Briggs, "Microtopographical roughness of shallow-water continental shelves," IEEE J. Ocean. Eng. 14, 360-367 (1989).

${ }^{25}$ K. B. Briggs, M. Zimmer, and M. D. Richarson, "Spatial and temporal variations in sediment compressional wave speed and attenuation measured at $400 \mathrm{kHz}$ for SAX04," in Proceedings of Boundary Influences in High Frequency, Shallow Water Acoustics, Bath, UK, September 2005.

${ }^{26}$ E. I. Thorsos and M. D. Richardson, "GuestEditorial," IEEE J. Ocean. Eng. 27, 341-345 (2002), p. 342, first column, lines 27-31.

${ }^{27}$ J. G. Proakis and D. G. Manolakis, Digital Signal Processing. Principles, Algorithms, and Applications. Third edition (Prentice-Hall, Englewood Cliffs, NJ, 1996), Chap. 11.1.1.

${ }^{28}$ E. G. Williams, Fourier Acoustics. Sound Radiation and Nearfield Acoustical Holography (Academic, London, 1999), Chap. 8.5, pp. 262-264.

${ }^{29}$ R. J. Urick, Principles of Underwater Sound. Third edition (Peninsula, Los Altos, CA, 1983), Chap. 8.13. 